Methods Support was offered by telephone, face-to-face home visits, in virtual groups or more recently in outpatients. Registered nurses and palliative care support workers hold their own caseloads. The service has provided advice and education for patients and families, enabled access to MDT, reduced social isolation, built relationships and introduced patients to hospice. STAR evolved using patient feedback and review of future model will include stakeholder engagement, cost effectiveness, activity levels, the broader community model and government guidelines.

Results Day hospice attendances, prior to the pandemic were, on average, 128 per month (2019-20) in contrast to 216 patient contacts per month 2020-21. With the same staffing model this provided a reduction in gross costs of over $£ 30$ per patient (Scheme $1 £ 101.16$ vs Scheme $2 £ 70.32$ ). Patient feedback is excellent - one patient stating 'STAR has been a lifeline in difficult circumstances when people needed to shield and were very isolated'.

Conclusions STAR has provided much needed support during a difficult period. Work is ongoing on the final community model at hospice although, through work so far, we know video training is as effective as face-to-face, patient satisfaction is high and the service is liked by the whole team but lack of peer support/social engagement is missed by patients and use of technology is a barrier for some.

\section{P-89 THE SURPRISINGLY POSITIVE OUTCOMES FOLLOWING THE COVID-19 RESPONSE WITHIN DAY HOSPICE}

Sarah Kerry. Ashgate Hospicecare, Chesterfield, Derbyshire

\subsection{6/spcare-2021-Hospice.107}

Background Day hospices, along with most other healthcare services changed dramatically in response to the COVID-19 pandemic, this included halting face-to-face services, stopping traditional day hospice models and in this case being left wondering what their role within the provision of specialist palliative care was. There was a period of grieving for what was seen as the loss of the day hospice service by staff members, colleagues, patients and their families. Despite the challenges, there have been some very positive outcomes from these enforced changes.

Aim The discontinuation of face-to-face contact within a traditional day hospice model enabled teams to evaluate their roles and services, leaving a blank canvas to allow development of more accessible and responsive services to meet changing needs during and after COVID-19.

Method The break in service allowed time for obtaining feedback from all users of the day hospice including patients, carers and professionals, enabling the development of user-led systems, processes and services.

Development and growth Feedback enabled the team to develop pilot programmes aimed to help meet the needs of service users. The pilots were then tested using the PDSA cycle, ensuring robust evaluation processes were embedded within each.

Results Pilots included the development of virtual services, a community hub, and MDT outpatient suite. These pilots are currently being evaluated and results pending.

Outcomes

- The opportunity to reflect and re-evaluate service aims.
- A flexible, empowered, and confident team.

- Developing an accessible, user-led specialist palliative care service using a quality improvement approach.

- Robust evaluations in place to measure the impact of pilot services.

\section{\begin{tabular}{|l|l} 
P-90 TRANSFORMATION OF THE LIVING WELL SERVICE \\
\hline
\end{tabular}}

Liesl Hopkins. St Richard's Hospice, Worcester, UK

\subsection{6/spcare-2021-Hospice. 108}

Background In one week the Living Well team completely changed the way they worked with patients and carers, from face-to-face to virtual support working from home (except essential home visits and essential reviews on the inpatient unit). Individual roles were adapted to meet the needs of patients and support of other hospice departments.

$\operatorname{Aim}(\mathrm{s})$

- The Living Well stepped model of care needed to continue to meet the needs of the patients and carers.

- To provide choice and empower patients to manage their own illness.

Methods

- 8 - 12 weeks virtual therapeutic programme via video or telephone call

- Virtual outpatient clinics via video call or telephone call.

- A wide range of therapeutic Living Well zoom courses including Men's Space, adapted Tai Chi, breathlessness, fatigue management.

- A wide range of drop-in groups via Zoom including nature therapy, art club and relaxation.

- 10 weeks empowerment webinar series including sleep hygiene, journaling, and self-care through bereavement.

- All patients had access to the Living Well virtual services leaflet and website resource page.

Results Attendances increased by $173 \%$ to 2,209 comprising; Therapeutic programme 442, Living well 413, clinics 677, Social/information groups 677 .

Conclusions We will offer a hybrid model of care (virtual and face-to-face) going forward, utilising the new space 'The Green'. This will enable the living well team to offer more choice and flexibility to patients and carers and empower patients, at any stage of their illness, to live well.

\section{P-91 POST COVID-19 COLLABORATIVE APPROACH TO SERVICE ADAPTATION BY THERAPY AND COMMUNITY DEVELOPMENT TEAMS}

Karen Bell, Christine Novelli. Isabel Hospice, Welwyn Garden City, UK

\subsection{6/spcare-2021-Hospice. 109}

Background Our service supports patients and carers to manage the challenges of living with a life-limiting condition; helps maintain independence and enhance quality of life, and gives patients the confidence to live as well as possible. Prior to COVID-19, we offered one-to-one therapy assessments, personalised programmes, group therapies and open-ended general wellbeing activities; our Compassionate Neighbours 
project supported those who were lonely and at risk of isolation. A full review of our services took place following the closure of all hubs, reduction in staff and enforced shielding of our patients, including the impact of social interaction for those vulnerable and isolated.

Aims To enhance the patient experience by offering episodes of clinical care, supported with a range of therapy interventions. Patients, their carers, and those bereaved are then invited to attend informal social meet-ups, or take part in wellbeing activities at new social hubs in community spaces to foster compassion and peer support. The hubs maintain a connection to the hospice until the point where clinical care might once again be needed.

Methods

- Developed a blended approach of online and face-to-face assessments.

- Introduced defined episodes of therapeutic care $(6,8$ or 12 week groups).

- Patient-centred programmes focus on achieving specific personal goals (Fettes, Ashford, Maddox, 2018).

- All care led by Integrated Palliative Outcome Scale (IPOS)

- Therapy to Compassionate Community (Compassionate Communities UK) caseload transition.

- Set up new social hubs.

Results 53 patients transitioned (Apr 20-May 21). Increased feedback from 'Views on care' to aid evaluation. Carer experience significantly improved.

Conclusions We adapted our service to bring an exciting solution towards enhancing the patient and carer experience by replacing 'discharge' with 'episode of care' and offering a transition from clinical therapy to community caseload for continued support and connection. This model has also enhanced our family support service.

\section{P-92 COVID-19 PRESSURE BEDS: RELIEVING THE DEMAND ON ACUTE BEDS BY TRANSFERRING INTO HOSPICE CARE}

Karen Walker. Ashgate Hospicecare, Chesterfield, UK

\subsection{6/spcare-2021-Hospice.110}

Background Throughout the COVID-19 pandemic, health and care systems were under immense pressure. The hospice made the decision to assist the local trust and admit palliative patients who had tested positive for COVID-19 into six pressure beds. Building on the partnership between the hospice and local trust, the beds were accessed by the trust through a new patient pathway of admission between October 2020 and March 2021.

Aim To achieve a timely, safe and appropriate transfer of COVID-19 criteria led patients from primary to hospice care.

Methods A new admission transfer criteria was introduced patients with non-complex palliative care needs who were COVID-19 positive. A service level agreement was established to enable a consistent and collaborative approach. The ward sisters held daily morning meetings to discuss identified admissions. A designated wing was established to care for COVID19 positive patients to prevent transmission.

Results A total of 40 admissions were taken since Nov. 2020. The transfer of acute patients to hospice care resulted in 369 saved bed days. Families were allowed precious time during the last days of life for $65 \%$ of patients in a calm and caring environment maintaining privacy and dignity for patients in their last days. Able to facilitate safe discharges home when patients recovered from the virus. Despite the implemented patient criteria, all patients transferred still required medical input due to their complex needs.

Conclusion Our findings demonstrate that working in partnership with the wider health and care system improves patient access to hospice care. Invaluable patient and family experience provides strong evidence for the continuation of the additional six beds. Admission criteria must be robust and flexible. We can restart the pathway if a third wave occurs and the hospice is further exploring how we can continue to support our local trust with the option of flexible winter pressure beds.

\section{P-93 SETTING UP A 'VIRTUAL WARD' WHEN THE INPATIENT UNIT CLOSES: LEARNING FROM CRISIS AND OPPORTUNITY}

Arjun Kingdon, Matthew Sweeting, Eva Lew. Farleigh Hospice, Chelmsford, UK

\subsection{6/spcare-2021-Hospice.111}

Background Farleigh Hospice provides palliative and end-oflife care to the people of Mid Essex (population c. 400,000), with a dedicated inpatient unit (IPU) and community services. Problem The first wave of the COVID-19 pandemic catalysed organisational change with creation of 'Locality Care Teams' and the upskilling of non-clinical members of staff. Subsequently, the IPU closed and staff were reassigned to Locality Care Teams in November 2021, mobilising the workforce to care for more patients. However, there was a resulting potential for some patient and carer needs to go unmet.

Intervention A community-based nurse-led 'Virtual Ward' was created in mid-December at rapid pace, to support people to die at home. A detailed SOP was developed, specifying the purpose of the Virtual Ward, eligibility criteria, and referral, assessment and discharge processes. This information was disseminated to all teams. Data were collected throughout the time of IPU closure.

Outcome Within a short time, the virtual ward was well-utilised (mean 8.6 admissions per month). Referrals to the Virtual Ward were largely appropriate with mean length of stay seven days, median three days. $80 \%$ of patients admitted to the Virtual Ward died as expected while under its care; none were admitted to acute care services. Informal feedback has been positive from referrers, patients and family caregivers.

Learning The temporary closure of the IPU could have caused patient and carer needs to go unmet. Instead, the rapid set up of the Virtual Ward, alongside the reassignment of IPU staff and arrangements with other regional hospices, has enabled the local population's end-of-life care needs to be well met. During the pandemic the greatest need for palliative care services has been in the community (Etkind, Bone, Lovell, et al., 2020; Costantini, Sleeman, Peruselli, 2020). Farleigh Hospice's reconfiguration of services has proven to be effective and responsive. There is a need for more robust data collection to evaluate outcomes particularly with regard to patient and family caregiver feedback. 